\title{
La enseñanza de la gramática en la escuela: lo que piensan, cómo la abordan y qué proponen las investigaciones brasileñas
}

\author{
Adriana Dickel \\ Universidad de Passo Fundo, Rio Grande do Sul, Brasil
}

(Artículo recibido el 1 de mayo de 2012; versión final recibida el 5 de mayo de 2012)

\begin{abstract}
En la década de 1980, en Brasil, se instala una polémica que se extiende hasta los días actuales: “¿se debe, o no, enseñar gramática en la escuela?”. Tal pregunta la plantean algunos sectores académicos en su crítica a la enseñanza tradicional de la gramática normativa. La suceden propuestas de manutención de la enseñanza de la gramática, vinculadas a la teoría de enunciación bakhtiniana y a teorías sociointeraccionistas y funcionalistas del lenguaje, presentes en los Parámetros Curriculares Nacionales y en los artículos aquí analizados. En este artículo, se pretende explorar, a través del estudio bibliográfico, tensiones y abordajes presentes en discusiones sobre la enseñanza de la gramática en la escuela, en las últimas décadas, en Brasil, y evidenciar que el encuentro entre la producción científica, didáctica y prácticas pedagógicas aún es una tarea que tiene que ser realizada por investigadores tanto del campo de la lingüística aplicada como de la educación.
\end{abstract}

\section{Introducción}

Este artículo proviene del proyecto de investigación "El conocimiento sobre el lenguaje escrito en el proceso de reflexión metalingüístico: la enseñanza de la gramática en los años iniciales de escolarización”, desarrollado por el Grupo de Estudios e Investigación sobre la Alfabetización - GEPALFA (Universidad de Passo Fundo/RS/Brasil). Situándolo en el ámbito de la investigación en la educación, más concretamente en la didáctica y metodología de la enseñanza, pretendemos analizar las aulas de lengua portuguesa y el papel de las situaciones de enseñanza en el proceso de adquisición de la lengua escrita y en el desempeño de los niños en relación con las elecciones lingüísticas implicadas en su uso. El diálogo sistemático con el campo de los estudios del lenguaje, nos orienta hacia la propuesta según la cual, los niños en la escuela deben encontrar las condiciones para aprender a considerar la lengua como un objeto que se puede manipular, sobre el cual se puede hablar y que es fundamental tener conocimientos explícitos. Desde esta perspectiva el sujeto puede transponer los límites establecidos por su mundo cultural e intervenir por medio de la lengua en los diversos contextos socioculturales en los cuales participa o va a participar. Para eso, el maestro tiene que exponer a sus alumnos a situaciones concretas de 
manejo de elecciones lingüísticas, y al mismo tiempo de distanciamiento en relación a esa misma lengua, con la intención de tomar conciencia del saber internalizado, del saber en uso y del saber posibilitado por otros recursos de la lengua aún no aprendidos.

La posibilidad que tenemos de operar sobre la lengua (por medio de reflexiones metalingüísticas), además de operar con la lengua, trae para la educación lingüística, concebida como

el conjunto de factores socioculturales que, durante toda la existencia de un individuo, le posibilitan adquirir, desarrollar y ampliar el conocimiento de/sobre su lengua materna, de/sobre otras lenguas, sobre el lenguaje de un modo más general y sobre todos los demás sistemas semióticos. (Bagno \& Rangel, 2005, p.63) ${ }^{1}$

una diversidad de desafíos y de posibilidades didácticas, con posibilidad de incrementar de modo sustancial el proceso de enseñanza-aprendizaje de una lengua.

En este sentido, se hace necesario abordar el papel de la enseñanza de la gramática en los diferentes niveles de educación básica, ${ }^{2}$ responsables del atendimiento de "las necesidades básicas del aprendizaje”, que comprenden a

tanto los instrumentos esenciales para el aprendizaje (tales como la lectura y la escritura, la expresión oral, el cálculo, la solución de problemas), como los contenidos básicos del aprendizaje (los conocimientos, habilidades, valores y actitudes) necesarios para que los seres humanos puedan sobrevivir, desarrollar plenamente sus potencialidades, vivir y trabajar con dignidad, participar plenamente del desarrollo, mejorar la calidad de vida, tomar decisiones fundamentales y continuar aprendiendo. (UNESCO, 1990, pp. 2-3)

Este es el tema del presente artículo. Atravesada por discusiones polémicas y por presupuestos no siempre esclarecidos, la temática de la enseñanza de la gramática aparece y desaparece, principalmente, al sabor de las críticas hechas a la inoperancia de la escuela de dotar a los sujetos de los instrumentos que les permitan aprender, sobrevivir y desarrollarse en una sociedad en constante transformación.

Es nuestro propósito, en este trabajo, sistematizar algunas de las principales contribuciones ofrecidas por intelectuales e investigadores brasileños sobre esta discusión. Nos orienta la expectativa de, a través de un estudio bibliográfico, congregar diferentes posiciones acerca del tema, elucidar las concepciones de la lengua y de la gramática que subyacen y reconstruir algunas propuestas para su enseñanza en la escuela. 
Para esto, inicialmente, revisaremos la historia de esta discusión en Brasil, focalizando nuestra reconstrucción en algunas obras que demarcaron posiciones acerca del tema, y que se volvieron una referencia para los estudios y definiciones curriculares posteriores. Después, analizaremos un corpus de artículos científicos publicados en los últimos cinco años en Brasil, que tienen como objeto la reflexión sobre prácticas de la enseñanza de la gramática en las escuelas y propuestas didácticas que vislumbran la superación del abordaje centrado en la gramática normativa, punto de críticas desde hace treinta años. Finalizaremos este artículo describiendo los retos a los que se enfrentan, actualmente en Brasil, los estudios sobre la didáctica de la lengua y, de manera especial, la enseñanza de la gramática.

\section{Marcos y polémicas sobre la enseñanza de la gramática en la escuela}

La tradición de la enseñanza de la lengua portuguesa en la escuela fue inaugurada en el siglo XVIII a través de la reforma hecha por el Marqués de Pombal (que concernió no solamente a Portugal, sino también a sus colonias, entre ellas Brasil). La reforma, según Bunzen (2011), se justificaba por la necesidad de garantizar la preservación de la lengua y de su diseminación a los pueblos dominados, siendo así la mayor responsable de la enseñanza obligatoria de la lengua portuguesa en Portugal y en Brasil.

No obstante, el estudio de la gramática de la lengua portuguesa, conjuntamente con la retórica, servía como recurso para el aprendizaje de la gramática del latín. Era esta la lengua necesaria para los estudios a nivel secundario y superior, ofrecidos por los jesuitas durante la época del Brasil colonial (Soares, 2004). Entre finales del siglo XIX y principios del siglo XX, la enseñanza de la gramática pasa a tener como objetivo el aprendizaje sobre el sistema de la lengua, actuando paralelamente a los estudios sobre retórica y poética, con miras al establecimiento de los preceptos sobre el hablar bien y después, en virtud de la pérdida de la importancia de la oratoria, sobre el escribir bien. Convivían en el aula los manuales de gramática y los manuales de textos seleccionados. En un contexto en el que tenían acceso a la escuela únicamente los hijos de las familias adineradas, tales conocimientos continuaban útiles y necesarios. 
A medida que las condiciones sociales y políticas se modifican, al inicio de la segunda mitad del siglo XX, se observa un cambio significativo en lo que se considera el contenido de la disciplina "Portugués". La democratización del acceso a la escuela, resultado de los movimientos reivindicatorios protagonizados por los grupos populares, permite el acceso a la escuela también a los hijos de los trabajadores. Eso produce necesidades y exigencias diversas de aquellas que hasta entonces no habían sido enfrentadas por las escuelas, repercutiendo, no solamente en el crecimiento del número de escuelas y de plazas, sino también en el número de docentes reclutados para la enseñanza y en la constitución de las disciplinas escolares.

En este contexto, el tratamiento de los contenidos de la disciplina "Portugués” se modifica profundamente:

gramática y texto, estudio sobre la lengua y estudio de la lengua comienzan a constituir una disciplina con contenido articulado: ahora es en la gramática donde se buscan elementos para la comprensión y la interpretación del texto, ahora es en el texto donde se van a buscar estructuras lingüísticas para el aprendizaje de la gramática. (Soares, 2004, p.167)

Esa fusión demarca una ruptura con la tradición jesuita y provoca modificaciones en los manuales didácticos, que pasan a ser más valorizados que en los periodos anteriores. Este recurso empieza a dividir con el maestro la tarea de planificar sus aulas, y a contener ejercicios de vocabulario, de interpretación, de redacción y de gramática.

Luego, en los inicios de la década de 1970, bajo la égida y en conformidad con la ideología del régimen militar instalado en 1964, todo el sistema educativo pasa por profundos cambios. Uno de ellos se hace notar en el ámbito de la enseñanza de la lengua portuguesa. Concebida por el régimen como instrumento de desarrollo, la disciplina pasó a tener como cuadro de referencia de análisis la teoría de la comunicación.

La concepción de la lengua como sistema, prevalente hasta entonces en la enseñanza de la gramática, y la concepción de la lengua como expresión estética, prevalente en la enseñanza de la retórica y de la poética y, posteriormente, en el estudio de los textos, son sustituidas por la concepción de la lengua como comunicación. Los objetivos pasan a ser pragmáticos y utilitarios: se trata de desarrollar y de perfeccionar los comportamientos del alumno como emisor y receptor de mensajes, a través de la utilización y de la comprensión de códigos diversos - verbales y no verbales. O sea ya no se trata más del estudio sobre la 
lengua o del estudio de la lengua, si no del desarrollo del uso de la lengua. (Soares, 2004, p.169)

En los libros didácticos disminuye el espacio anteriormente dado al estudio de la gramática, se extiende la selección de textos para otros géneros además de los literarios y se incluye el tratamiento de textos no verbales y del lenguaje oral.

El enflaquecimiento del régimen militar, que culmina con su disolución en 1985, conjuntamente con las nuevas teorías desarrolladas en el ámbito de las ciencias lingüísticas, trae consigo una fuerte reacción a ese modelo. En el ámbito de la formación de maestros, desde la lingüística, la sociolingüística, la psicolingüística, la lingüística textual, la pragmática y el análisis del discurso empiezan a aparecer análisis sobre la enseñanza de la lengua.

Comienza a ocupar las discusiones la problemática sobre las variaciones lingüísticas -evidentes con la ampliación del acceso de los grupos populares a la escuela y con la heterogeneidad lingüística presente en las aulas- y la pertinencia y observancia de la modalidad del padrón ${ }^{3}$ de la lengua como objeto de enseñanza. Otro elemento que atañe fuertemente la enseñanza de la gramática en la escuela proviene de los estudios descriptivos de la lengua portuguesa, los cuales se remontan a otras concepciones de la gramática que remiten, por ejemplo, a la gramática de la lengua hablada. Por fin, la lingüística textual impone una ampliación del campo en los estudios de la gramática por medio de la introducción de una nueva manera de tratar el texto. El cuadro de innovación teórica recibe un impulso con la presencia en el ámbito académico de la pragmática, de la teoría de la enunciación y del análisis del discurso. Soares (2004) además destaca, en el escenario posdécada de 1980, la presencia de los estudios sobre la historia, la sociología y la antropología de la lectura y de la escritura.

Este intento de aproximar los estudios académicos del mundo escolar ocurrió concomitantemente con lo que se conoció como "la crisis de la escuela". Organizada para atender las necesidades de los grupos privilegiados de la población, la escuela (y su currículo) se vio invadida por un amplio sector de la población, que antes no tenía acceso a las aulas, y pasa a mostrar sus límites para atender a esta nueva realidad, expuestos en los índices de reprobación y de evasión escolares. Este cuadro hace surgir un discurso 
contundente de crítica hacia la enseñanza tradicional y a su papel en la producción del fracaso escolar.

Según Angelo (2005), en este contexto de crisis, la enseñanza de la lengua portuguesa tuvo su parte de culpa, una vez que los índices de retención se conglomeraban en la primera serie del ensino fundamental y que las competencias de lectura y de escritura de los estudiantes de niveles avanzados eran precarias. A su vez, los lingüistas asumen la tarea de criticar los modelos que orientaban la enseñanza de la lengua en la escuela, principalmente a aquellos pautados en la concepción normativa de la lengua, representante de una única variedad de la lengua, la padrón, y una única modalidad de la lengua, la escritura (Pietri, 2010). Además, comienzan las críticas al tiempo invertido en el estudio de esta variedad, en un contexto de heterogeneidad lingüística, que decididamente no repercutía sobre el desempeño de los estudiantes en la lectura y la producción de textos.

\section{Algunas referencias de la polémica sobre (no) enseñar gramática en la escuela}

Como se puede percibir, se había creado el ambiente intelectual para el surgimiento de la controversia: “¿se debe o no enseñar gramática en la escuela?” Desde los años 1980 hasta nuestros días, se observa una gran cantidad de producciones sobre el asunto. De ellas, destacaremos tres obras -Geraldi (1984a), Possenti (1996) e Perini (2010)- que a nuestro entender son representativas de esta polémica, y al mismo tempo explicitan posiciones distintas con relación a esta crisis.

De Geraldi (1984a) consta un conjunto de artículos ampliamente debatidos en los foros académicos, y además de eso, en los sectores responsables por las definiciones curriculares. Los textos presentan la crítica a la enseñanza tradicional de la lengua y la reivindicación por la lingüística como base para una propuesta de enseñanza que "resulte positiva”. Uno de ellos combate la enseñanza de la gramática en la escuela, identificándola como “una de las siete plagas de la enseñanza del portugués” (Faraco, 1984).

Se destacan por su preocupación de brindar elementos para proyectar la enseñanza sobre nuevas bases dos artículos de Geraldi (1984b, 1984c). En ellos existe una percepción de la necesidad de la escuela, en un contexto de diversidad lingüística, “dar oportunidad o 
dominio al dialecto padrón”, pero no mediante las estrategias adoptadas por la enseñanza tradicional, o sea, mediante "ejercicio de reglas e hipótesis de análisis de problemas que los mismos especialistas no están seguros de resolver" o por medio de “conceptos y metalenguajes con los cuales se habla sobre la lengua, se presentan sus características estructurales y de uso". Según él, esos conocimientos se deberían hacer presentes en la enseñanza de la lengua "a penas subsidiariamente”, solo "cuando la descripción de la lengua se impone como medio para alcanzar un objetivo final de dominio de la lengua, en su variedad de padrón” (Geraldi, 1984b, pp. 46-47). En su crítica, el autor defiende la necesidad de abrir espacios para prácticas de análisis lingüísticas, realizadas con base en los textos producidos por los alumnos, mediante los cuales la tarea del maestro sería seleccionar los problemas para la realización de actividades de re-escritura.

Esta obra influenció de manera importante las reformas curriculares de diversas provincias brasileñas, sirviendo, inclusive, como se verá más adelante, de referencia para la elaboración de los Parámetros Curriculares Nacionales (a partir de ahora PCN), producidos a finales de la década de 1990, por iniciativa del Ministerio de Educación.

Possenti (1996), en “¿Por qué (no) enseñar gramática en la escuela?”, se inserta en el debate sistematizando algunos descubrimientos “científicos” en el dominio de la lingüística, bastante sólidos, aunque no suficientemente difundidos, sobre lo que es una lengua y su proceso de aprendizaje. El autor, así como otros en esos años, defiende que el dominio activo de la lengua prescinde del estudio de la gramática y que enseñar una lengua no es lo mismo que enseñar gramática. Para él, la opción de enseñar gramática y el tiempo destinado a esta actividad, en detrimento del trabajo con el texto (lectura y producción), exponen las concepciones sobre el papel de la lengua en una sociedad de contrastes y los objetivos atribuidos a la escuela. Partiendo de este presupuesto, Possenti (1996, p.60) ofrece "una propuesta de enseñanza de la gramática destinada específicamente a quien tiene como utopía alumnos que escriban y lean, también en situaciones relativamente precarias, esto es, antes de la alteración de las condiciones sociales actuales”. Para abordarla, presenta tres concepciones de gramática - la normativa, la descriptiva y la internalizada - y afirma que la enseñanza de la gramática en la escuela aún es un objetivo válido desde el momento 
en que se invierta la prioridad que normalmente es dada a la gramática normativa, en primer lugar a favor de la gramática internalizada, y, en segundo lugar, de la gramática descriptiva.

Para él, la escuela debe ofrecer condiciones para que el "alumno pueda dominar efectivamente el mayor número posible de reglas, esto es, que sea capaz de expresarse en las más diversas circunstancias, según las exigencias y convenciones de estas circunstancias”. Por tanto, no cabe sustituir la enseñanza de una variedad por otra, sino “crear las condiciones para que los alumnos aprendan también las variedades que no conocen, y con las cuales no tienen familiaridad, incluida la que es peculiar en una cultura más elaborada” (Possenti, 1996, p.83).

La posición de Possenti, observada en trabajos de otros lingüistas como Soares (1989) y Travaglia (1995), dota a la polémica sobre la enseñanza de la gramática de una perspectiva política, situándola en un contexto de lucha por la transformación social intensa en los años 1980 y 1990 -por medio también de la conquista del derecho a una educación de calidad para todos.

Perini (2010) atribuyó a la enseñanza de la gramática una connotación distinta. Para él, la gramática debe ser incorporada al currículo por razones culturales. Esta posición está expuesta en su obra Para uma nova gramática do Português (1985), y recientemente retomada en Gramática do português brasileiro (2010), un importante estudio sobre la gramática del portugués hablado. Así como Possenti (1996), Perini (2010) denuncia la inconsistencia de la creencia según la cual el estudio de la gramática debe llevar a la apropiación de la variedad padrón de la lengua y al incremento de las capacidades de leer y escribir.

Según Perini, la escuela exige que el conocimiento gramatical sea aplicable. Esta condición de aplicabilidad no se exige a los conocimientos de otros ámbitos como por ejemplo la biología, la física o la geografía. Para el autor, la gramática es una disciplina científica, esto es,

Así como la biología estudia a los seres vivos (...) y la química estudia a los elementos y sus combinaciones, la gramática estudia un aspecto del lenguaje (su 
estructura formal y semántica) - un fenómeno tan presente en nuestras vidas como los seres vivos o los elementos químicos. (Perini, 2010, p.35)

Como señala Perini, el conocimiento de una lengua es parte del conocimiento del mundo, accesible a la observación a través del comportamiento y de los juicios de los hablantes. La gramática “es una disciplina científica, pues tiene como finalidad el estudio, la descripción y la explicación de los fenómenos del mundo real” (Perini, 2010, p.37). Esta no agota el estudio de la lengua, ni de la comunicación humana, pero toma parte de ambas una vez que "ninguna sociedad humana prescinde de la comunicación, ninguna existe sin una lengua, y todas las lenguas tienen gramática” (Perini, 2010, p.19).

Como disciplina científica, ella es parte de la formación científica de los alumnos, algo indispensable para la vida en pleno siglo XXI, lo que justifica su presencia independientemente de los resultados que su estudio produce en las habilidades de leer o escribir. El autor parte de la idea que la ciencia hoy es fuente de poder y, por tanto, la formación científica es un elemento fundamental de la educación en los días actuales. Aunque no todos los alumnos se conviertan en científicos, el sujeto alfabetizado científicamente se vuelve capaz de analizar y de emitir su opinión, de tomar posición sobre las diferentes situaciones que afectan la vida en la sociedad.

El ciudadano de hoy, por lo menos en las democracias, participa de las decisiones importantes de su comunidad. Y una de las guías que nos orientan en esas decisiones es nuestra alfabetización científica. El analfabeto científico es una criatura indefensa, un juguete de opinión y de los intereses claros y oscuros de otras personas. (...) La democracia, para sobrevivir, necesita extender la educación política, humanística y científica para toda la población. (Perini, 2010, pp. 31-32)

En esta perspectiva, la ciencia "no es un cuerpo de conocimientos y resultados; es un método para obtener conocimientos y resultados” (Perini, 2010, p. 32). El maestro de matemáticas no enseña (y hace memorizar) los resultados de las multiplicaciones, por ejemplo, pero enseña el proceso para llegar al resultado. Ya en el caso de la enseñanza de la gramática, la lógica se encuentra no raras veces invertida, tocándole al alumno repetir resultados sin adentrarse al método que lo llevó a su obtención. La enseñanza de la gramática que se practica en las escuelas contribuye al analfabetismo científico por trabajar 
con datos ficticios, evitar las dudas y fortalecer "la creencia sin crítica en las doctrinas aprendidas, pero no justificadas” (Perini, 2010, p. 35).

En las clases de gramática no se aprende gramática, ni siquiera se estudia gramática. No es de espantar que los alumnos (y los maestros, que también son víctimas del sistema) no sepan gramática, no se interesen por la gramática y, para resumir todo, detesten la gramática. (Perini, 2010, p. 34)

Para romper este ciclo e instaurar la curiosidad científica dentro del estudio de la lengua, el autor considera fundamental no solamente aprender ciencia sino hacer ciencia. Para él, una disciplina científica no es solamente una fuente de informaciones sobre el mundo, sino "un campo de ejercicio del pensamiento independiente, de observación imparcial y cuidadosa, de respecto a los hechos - habilidades preciosas, cada vez más necesarias, pero que brillan por su ausencia en la enseñanza tradicional de la gramática” (Perini, 2010, p. 39). En este sentido, sugiere que se trabaje la gramática, primeramente, abandonando la idea que su estudio mejora las habilidades en la lengua escrita y que la gramática es un sistema plenamente conocido y listo, lo que permitirá establecer nuevos propósitos para la disciplina; en segundo lugar, asumiendo una actitud científica con relación al lenguaje, dando lugar a dudas y justificaciones para las afirmaciones hechas y para los cuestionamientos; y, por último, permitiendo la observación, la manipulación, el estudio de hechos de la lengua, con el objetivo de formular hipótesis al respecto de cómo ésta funciona.

\section{La concepción de la enseñanza de la gramática en los PCN}

Con la aprobación de la Ley de Directrices y Bases de la Educación Nacional en 1996, en sustitución de la de 1971, promulgada en pleno régimen militar, se establece una base común nacional que dará referencia a las propuestas curriculares en todos los niveles en el sistema de enseñanza. Hasta entonces, con excepción de la enseñanza superior, las definiciones curriculares eran responsabilidad de cada provincia.

Publicados entre 1997 y 1998, los PCN incorporan la crítica a la escuela, que atraviesa la década de 1980 y alcanza la siguiente. La crisis de la escuela y, como resultado, el fracaso escolar tienen como respuesta un documento con un conjunto de orientaciones 
fundamentadas en las conquistas científicas en las diferentes áreas del conocimiento y una tentativa de aproximación entre la escuela y la academia.

No es nuestra intención profundizar en el análisis de este fenómeno, pero nos interesa destacar algunas propuestas respecto a la enseñanza de la gramática en la escuela y la configuración que los PCN dan sobre esta cuestión. ${ }^{4}$ Los PCN (Brasil, 1998) en el área de la lengua portuguesa ${ }^{5}$ incorporan discursos y aproximaciones teórico-metodológicas que eran actuales en el momento de su elaboración. Incluyen conceptos tales como: condiciones de producción, género de texto, texto, textualidad, discurso, reflexión sobre la lengua, variación lingüística, competencia discursiva, lingüística y estilística, entre otros, que habían sido puestos en evidencia por los estudios lingüísticos de aquel momento. Conciben el lenguaje como actividad discursiva y el texto como unidad de enseñanza, atribuyendo a las diversas prácticas del lenguaje un papel central en el trabajo pedagógico. Son ejes articuladores de los contenidos de la lengua portuguesa el uso de la lengua oral y escrita y la reflexión sobre la lengua y el lenguaje. Los PCN incluyen, en el eje “uso”, los contenidos curriculares de la práctica de escuchar textos orales y la lectura de textos escritos y la práctica de producción de textos orales y escritos, y, en el eje "reflexión”, la práctica del análisis lingüístico.

Entre las tres obras a las que hicimos referencia en el punto anterior (Geraldi, 1984a, Possenti, 1996; Perini, 2010), la que más repercutió en la elaboración de los PCN fue, sin duda, la de Geraldi (1984a). El concepto de análisis lingüístico expuesto originalmente por este autor, es usado como sustituto de la expresión “enseñanza de la gramática”. Esta sustitución tiene como finalidad asegurar que el estudio de la lengua no ocurra desvinculado de las prácticas sociales del lenguaje, de la producción y recepción de los diferentes géneros de textos. Los elaboradores de los PCN pretenden así desplazar el enfoque de la enseñanza de la lengua hacia el enfoque del uso del lenguaje y de la reflexión sobre este uso, o sea, para los procesos comunicativos en curso en las diferentes situaciones sociales, lo que denota su clara orientación socio-interaccionista, apoyada en la presencia ginebrina entre los consultores del Ministerio. 
Según Geraldi (1993, 1996), cuya base teórica se sustenta en la teoría de enunciación bakhtiniana, la práctica del análisis lingüístico incluye actividades que todo hablante puede hacer con la lengua: actividades lingüísticas, epilingüísticas y metalingüísticas ${ }^{6}$. Las primeras se refieren a un cierto tipo de reflexión, que podría decirse casi “automática”, que ocurre sin que el flujo de la interacción entre sujetos necesite ser suspendido. Las actividades epilingüísticas se refieren a la evaluación que todo hablante realiza de los recursos expresivos que utiliza y que son puestos a su disposición, lo que acarrea una interrupción en la corriente de la comunicación que se mantiene hasta que los enunciados asuman las formas de las intenciones. Las últimas son actividades "que toman al lenguaje como objeto, no ya como reflexión vinculada al propio proceso interactivo, sino que exigen el uso sistemático de un metalenguaje, con el cual los sujetos hablan sobre la lengua” (Geraldi, 1993, pp. 20-26), analizándola por medio de conceptos, clasificaciones, etc.

Como se trata de actividades accesibles a todas las personas y no solamente a especialistas, en los PCN, ese abordaje asume connotaciones de estrategia didáctica para el análisis lingüístico a disposición del maestro.

La actividad más importante, pues, es la de crear situaciones en que los alumnos puedan operar sobre el propio lenguaje, construyendo, poco a poco, en el curso de varios años de escolaridad, paradigmas propios del habla de su comunidad, observando las similitudes, regularidades y diferencias entre las formas y los usos lingüísticos, construyendo hipótesis sobre las condiciones contextuales y estructurales en que ocurren. Es a partir de lo que los alumnos consiguen intuir en este trabajo epilingüístico, tanto sobre los textos que producen como sobre los textos que escuchan o leen, que podrán hablar y discutir sobre la lengua, registrando y organizando estas intuiciones: una actividad metalingüística que implica la descripción de los aspectos observados por medio de la categorización y del tratamiento sistemático de los diferentes conocimientos construidos. (Brasil, 1998, p. 28)

No obstante esos presupuestos, predomina en los PCN un discurso que enfatiza mucho más lo que no se debe enseñar y lo que no se debe de hacer que lo que debe constituir el planificación del maestro y como él debe proceder para que las prácticas tradicionales ampliamente combatidas en el documento (y fuera de él) no sean reproducidas. Algunos enunciados ilustran esta afirmación: "Debe tenerse claro, en la 
selección de los contenidos de análisis lingüístico, que la referencia no puede ser la gramática tradicional”, “lo que debe ser enseñado no responde a las imposiciones de organización clásica de contenidos en la gramática escolar”, “el modo de enseñar, por su parte, no reproduce la clásica metodología de definición, clasificación o ejercitación”. (Brasil, 1998, pp. 28-29).

Por tanto habitan en el documento una exposición de las concepciones defendidas por las esferas académicas y un discurso prescriptivo al revés, eso es, que denuncia los errores de las prácticas pero pocos datos ofrece sobre el camino para una enseñanza “correcta”. Sobre el carácter de propaganda de los PCN, Marinho (2003) presenta un análisis interesante y con el cual concordamos. En su estudio sobre las estrategias textuales y enunciativas adoptadas por los PCN, la autora observa una ambigüedad en el documento: por un lado ocurre un "apagamiento de la autoría”, en virtud de la omisión de grande parte de las referencias teórico-metodológicas a lo largo de la exposición (inclusive aquellas citadas por nosotros anteriormente), por otra parte, el lector se enfrenta con la presencia profusa de ciertas bibliografías, algo inusitado en un documento de esa naturaleza. Según Marinho (2003), antes de ser un documento oficial, los PCN pueden ser concebidos como un texto de divulgación científica, o como un texto académico. El hecho, independientemente de la opción que hiciéramos para definirlo, es que por él hablan muchas “voces”, principalmente aquellas que poseen una hegemonía del pensamiento sobre la enseñanza de la lengua portuguesa en la escuela.

\section{Actualizando la discusión: las investigaciones recientes y la enseñanza de la gramática}

Como vimos, el debate sobre la enseñanza de la gramática en la escuela tiene dos posiciones convergentes: la enseñanza tradicional de la gramática no eleva las condiciones de uso y de comprensión sobre la lengua por parte de los aprendices; y es la escuela la que debe abordar ese campo de conocimiento sistemáticamente. Ya los objetivos y los desdoblamientos de esos presupuestos tienden a ser diversos.

Si la lengua es un legítimo objeto de enseñanza, ¿qué lugar tiene en el proceso de enseñanza-aprendizaje el estudio y la reflexión sobre la gramática de la lengua? ¿Si la 
enseñanza de la gramática no asegura adquisiciones que se traspongan para el uso adecuado y eficiente del oral o escrito de la lengua, qué justificaría su presencia en el currículo? ¿Qué áreas del conocimiento contribuyen para la explicitación de esa problemática y para la formulación de sus respuestas? ¿Cómo las producciones científico-académicas más recientes enfrentan las cuestiones sobre qué gramática enseñar, con qué objetivo y de qué forma?

Sin la intención de contestar a todas esas preguntas, nos vemos en la necesidad de identificar cómo ha sido tratada la inserción de la gramática en la escuela, en los trabajos académicos más recientes, verificando en qué términos aquellas premisas para las cuales convergen las discusiones se articulan con el trabajo efectivo en el aula.

Para esto hicimos una búsqueda en la base de datos Scielo, mediante el recurso de las siguientes palabras clave: gramática, enseñanza de la gramática, reflexión lingüística, metalenguaje, reflexión metalingüística, lengua portuguesa, lengua materna, enseñanza, aprendizaje, análisis lingüístico, utilizadas de manera combinada o aislada. El resultado nos llevó a 67 artículos vinculados a revistas del área de la lingüística y de la educación, en el periodo 2005-2011. Después de la lectura de los títulos y de los resúmenes de los artículos, fueron seleccionados los trabajos que se referían a la enseñanza de la gramática (o al análisis lingüístico, de acuerdo con los PCN) investigada desde un punto de vista didáctico. De esa segunda selección resultaron 18 textos. Con la lectura de los artículos, se hizo necesaria otra tría, porque en la exposición la expectativa de los resúmenes no se cumplía. De esta manera se constituyó un corpus de 6 artículos enfocados en la problematización de prácticas de enseñanza o de innovaciones relativas al trabajo con la gramática. Realizados entre 2010 y 2011, 4 de estos fueron publicados por la Revista Brasileña de Lingüística Aplicada (Universidad Federal de Minas Gerais), 1 por la Revista Trabajos en Lingüística Aplicada (Universidad Estadual de Campinas) y 1 por la revista Educación y Investigación (Universidad de São Paulo).

Podemos observar que la circulación de trabajos sobre la enseñanza de la lengua portuguesa, además de aquellas que constituyen nuestro corpus, se da, tanto en el área de educación, como en el área de la lingüística, fundamentalmente de la lingüística aplicada, 
encontrándose dispersa entre estas áreas. Esa constatación reiteró una sospecha anterior: la ausencia de un campo como la didáctica de la lengua, para el cual puedan confluir trabajos de esta naturaleza y temática. Tal vez una de las razones sea el hecho de que en Brasil la formación profesional para la carrera de maestro en los años iniciales de la enseñanza fundamental esté a cargo de los cursos de la Licenciatura en Pedagogía y para enseñar en los años finales y en la enseñanza media la responsabilidad corre a cargo de los cursos de la Licenciatura en Letras.

Otro elemento que destacó fue el número restringido de trabajos con el enfoque que buscábamos. Esto puede indicar, como una primera hipótesis, que las prácticas didácticometodológicas en el área de la enseñanza de la gramática no les afectan mucho a los investigadores brasileños, los cuales en su mayoría, como observamos, reportan problemas de fundamentación y de investigación básica. Por otra parte, la segunda hipótesis es que tal vez eso acontezca para que el estudio de la gramática, en el contexto de las políticas curriculares actuales, deje espacio a las producciones didáctico-metodológicas enfocadas en las prácticas de lectura y de producción de textos, ya que ellas se centran, muy especialmente, en el trabajo con los géneros textuales. Este es el objeto de estudio de la mayor parte de los artículos escogidos durante la primera parte del proceso de selección descrito anteriormente. Tanto una hipótesis, como la otra, solamente podría ser investigada en el caso de que superáramos la fase exploratoria de nuestro estudio, y de que ampliáramos el universo de investigación hacia otros campos, tales como el Banco de Tesis y Disertaciones del Capes (Coordinación de Perfeccionamiento del Personal del Nivel Superior), los anales de los eventos consolidados en el campo de la lingüística y de la educación, las revistas científicas que no se encuentran en la base Scielo.

Ahora, procederemos con la descripción de los documentos con base en dos categorías: la crítica a la enseñanza de la gramática y la incorporación de nuevos enfoques teóricos a las prácticas de la enseñanza de la gramática, sus convergencias y divergencias. Este último recibirá más atención ya que volvemos nuestra atención a los trabajos que enfrentan el reto de abordar la gramática como objeto de enseñanza de manera que supere el abordaje tradicional. Emplearemos los siguientes códigos para referirnos a los trabajos 
que constituyen nuestro corpus: para Aparício (2010), T1; para Brito (2011), T2; para Cerqueira (2010), T3; para Fairchild (2010), T4; para Gonçalves, Saito \& Nascimento (2010), T5; y para Silva (2010), T6.

\section{Una crítica hacia la enseñanza tradicional de la gramática y la incorporación de otras concepciones y de nuevos enfoques teóricos}

Los autores de los textos seleccionados realizan sus trabajos en un contexto de críticas hacia la enseñanza tradicional de la gramática y de la búsqueda de referencias y de justificaciones para nuevas investigaciones en las contribuciones provenientes de los estudios lingüísticos y de las propuestas oficiales (PCN). Las características atribuidas a este tipo de enseñanza no difieren de aquellas reconstruidas en los tópicos anteriores. Se menciona, para caracterizarlo, el énfasis en la descripción y clasificación, en el metalenguaje, en la prescripción, en los determinados temas gramaticales cuyo abordaje se repite año tras año, y la tendencia de separar el estudio de la lengua como sistema del estudio de su funcionamiento en las condiciones efectivas en las cuales se realiza.

En T6, un aspecto está puesto en relieve. La autora parte del principio de que el debate académico expone la crítica de la enseñanza tradicional de la gramática, pero no produce resultados consensuales sobre lo que debería ser el objeto de estudio en la clase de portugués, dando origen a un "clima de desaliento" entre los maestros. Reconoce el esfuerzo de Geraldi (1984b; 1984c) pero afirma que su abordaje no constituye, aún, una tendencia dominante en la enseñanza de la lengua en el país.

T5 también considera que, a pesar del “viraje pragmático o comunicativo”, los contenidos de las clases enfocados en la gramática normativa resisten al cambio. Ante esto las autoras proponen que la perspectiva socio-interaccionista (Bronckart, 1999; Dolz \& Schneuwly, 2003) y la teoría de la enunciación bakhtiniana sean asociadas a la perspectiva funcionalista, una vez que ésta proporciona análisis lingüísticos que parten de la concepción de la lengua como "una estructura maleable, sujeta a las presiones de uso y constituida de un código no totalmente arbitrario” (Gonçalves, et al., 2010, p. 1004). Para comprobar la viabilidad de trabajar con los géneros discursivos desde una perspectiva funcionalista, las autoras presentan un análisis de textos del género propaganda 
institucional, extraídos de una edición especial de la revista Veja (uno de los semanales de mayor difusión en Brasil). La exposición se centra en el análisis descriptivo de cinco textos, mediante los cuales son presentadas las relaciones entre los sistemas discursivos, semánticos y gramaticales, articulados en la interacción productor/lector situados en un contexto de producción/recepción. Para ellas, esta es una práctica posible para ser realizada en la clase y que permite unir el trabajo realizado y el trabajo prescrito.

\section{Las prácticas de enseñanza de la gramática: convergencias y divergencias}

Un tema común en cuatro trabajos $(\mathrm{T} 1, \mathrm{~T} 2, \mathrm{~T} 3, \mathrm{~T} 6)$ es la relación en el aula entre los principios de una enseñanza pautada por el abordaje normativo de la lengua y una enseñanza innovadora, enfocada en el discurso del cambio presente en las producciones académicas y en los documentos oficiales.

T1, T6 y T2 se dirigen a los maestros, tanto de formación continua como de formación inicial. En T1, la autora aborda la comprensión que los maestros tienen de este contexto de cambio, la incorporación que hacen de su discurso y los cambios que efectivamente hacen en sus prácticas. Se trata de una investigación cualitativointerpretativa de naturaleza etnográfica. El corpus es un conjunto de clases grabadas por siete maestras de escuelas públicas, que enseñan en los últimos años del ensino fundamental. El análisis de las clases evidencia cómo construyen el objeto de enseñanza y qué estrategias utilizan las docentes. Constata, en muchas de ellas, el esfuerzo de llevar a los alumnos a la observación y a la reflexión de determinadas categorías y funciones gramaticales a través de textos, fragmentos de textos y oraciones. No fueron observadas técnicas en las que la palabra es la unidad de análisis o estrategias de identificación y clasificación de categorías gramaticales. Las maestras reconocen los límites de la enseñanza tradicional de la gramática, y demuestran dificultad en percibir lo que puede realizarse en el ámbito de las prácticas de reflexión lingüística. La autora concluye que las innovaciones, implicadas en la tarea de realizar lo que los documentos oficiales consideran como tratamiento didáctico del objeto de enseñanza, presentan los siguientes trazos comunes:

- Desarrollan un tratamiento lingüístico solamente con categorías de la gramática tradicional, aunque las demandas de innovación proponen el trabajo 
con otras categorías, como las de la gramática funcional y las de la lingüística de texto, por ejemplo;

- Para el estudio de las categorías de la gramática se valen de modos de descripción/análisis diseminadas por la lingüística, focalizando, sobre todo, en la dimensión de la lengua, y aún, de la dimensión morfosintáctica. (Aparício, 2010, p. 904)

Resulta de su análisis una concepción de la enseñanza de la gramática como un trabajo para el cual convergen varias lógicas y temporalidades y que se realiza en un colectivo (aula, escuela). Para la autora, la clase de gramática es un espacio dinámico, donde no existen fronteras entre lo innovador y lo tradicional, pero una mezcla en el que, en términos académicos y científicos y conforme las determinaciones oficiales, es concebido como excluyente.

En T6, el problema de investigación es muy semejante al de T1. La autora se propone entender como los maestros se comportan delante de los enfrentamientos entre la tradición gramatical escolar y los discursos que intentan superarla, por medio de la investigación de la práctica de dos maestros de escuelas públicas que trabajan en los últimos años del ensino fundamental. Con la misma aproximación metodológica, la investigadora reitera algunas de las conclusiones de T1 y afirma que resulta de la tentativa de la construcción de la identidad profesional de los docentes, en este contexto de cambio, reflexiones que se caracterizan por lo que ella denomina "solidaridad de nociones teóricometodológicas”, provenientes de la tradición gramatical y de la teoría lingüística. Esta solidaridad, según la autora, puede ser observada en tres comportamientos:

1) variación de perspectivas teóricas para abordar un mismo contenido; 2) adopción de posturas diferentes por parte del docente, conforme al contenido que se está enseñando; y 3) el abordaje de un mismo contenido por cuadros referenciales diversos persiguiendo objetivos diferentes. (Silva, 2010, p. 961)

En T2, el objetivo de analizar la confluencia entre las concepciones tradicionales de gramática y los demás modelos que permiten acoger la enseñanza de la lengua lleva la autora al ambiente de formación inicial de los docentes, precisamente, en el curso de Letras. A través de cuestionarios, se les pregunta sobre la necesidad de enseñar la gramática normativa (GN), los estudiantes revelan una concepción de la gramática como una “concretización perfecta y auténtica” de la lengua. Según ellos, la condición para ser 
maestro de portugués es el dominio de la GN. En su análisis, la investigadora utiliza las referencias teórico-metodológicas del análisis del discurso de la línea francesa y concluye que la gramática es para los futuros maestros algo que no tiene sentido, que no tiene historicidad, y cuyo aprendizaje necesita de un maestro que los motive para aprender por medio de una “pedagogía interesante, dialógica y democrática”. Esta perspectiva, según la autora, se confronta con un discurso de cambio, característico de los años de 1980 y 1990, y que aparece en los comentarios, hechos por los alumnos, sobre el papel del maestro de lengua. Según los entrevistados, el docente debe respetar el lenguaje del alumno y posibilitar la apropiación de la norma culta por los grupos pobres de la población, es decir la enseñanza respetuosa de la norma padrón. En cierta medida, las conclusiones de T2 van en la dirección de los dos trabajos anteriores: en el trayecto de su formación, los sujetos no sustituyen un conocimiento por otro, si no los sobreponen, los entrecruzan.

El estudio presentado por T4 es impulsado por un caso, construido con base en situaciones vividas por el autor como supervisor de prácticas profesionales en el curso de Letras. En un aula, los alumnos realizan una interpretación inusitada de la palabra "rataria” (enjambre de ratas), utilizada por Monteiro Lobato en una de sus fábulas (Lobato, 1994). Fairchild (2010) detalla una serie de posibles acciones que pudieran ser desarrolladas a partir de las respuestas dadas por los alumnos. Con esto, él pretende exponer de modo práctico a lo que llevarían los postulados teóricos que orientan actualmente en la enseñanza de lengua portuguesa.

El autor denuncia que, en las interacciones con los estudiantes, los errores son concebidos frecuentemente como un déficit, y no como aspectos que se deben estudiar/analizar/explorar para comprender los procedimientos de construcción de conocimientos y que ofrecen oportunidades para que el maestro se incluya en la palabra del alumno. Delante de las críticas hechas a la enseñanza tradicional, el autor, siguiendo el ejemplo de T6, indaga sobre qué propuestas alternativas son ofrecidas a los maestros y qué conocimientos necesitan para desarrollar una enseñanza que se oriente por otras propuestas. Según él, el maestro necesita de conocimientos técnicos y de un repertorio teórico que le proporcione condiciones de explicar el trabajo lingüístico que se exterioriza en los errores, 
o de aprovechar el error para otras actividades y reflexiones. Además, precisa elaborar una especie de capacidad que le permita lidiar tácticamente con lo inusitado, de modo que pueda movilizar los conocimientos sobre el lenguaje adecuada y productivamente. Ambos son fundamentales en la enseñanza y no hay cómo sustituir un tipo de conocimiento por el otro.

Respecto a los conflictos que se crea entre las teorías profesadas por los libros y las teorías que emergen de las propuestas de trabajo que estos libros contienen, T3 analiza cuatro obras orientado por la hipótesis según la cual en los libros didácticos se hacen presentes ejercicios y actividades que remiten, respectivamente, a una concepción tradicional de la enseñanza de la lengua y a una práctica que traspasa las preocupaciones gramaticales y se vuelve a la utilidad de ese conocimiento en la vida de los alumnos. Cerqueira (2010) recorre a las áreas de la lingüística, pedagogía y psicología para sistematizar la diferencia entre los conceptos de ejercicios y de actividades. Según ella, las actividades permiten realizar las propuestas de los PCN, en la medida que prevén el análisis lingüístico y las situaciones de reflexión epilingüístico y metalingüístico. El ejercicio está al servicio de la enseñanza de la gramática normativa, una vez que lleva al alumno a operar con la lengua, partiendo de un estímulo provocado por el maestro y llegando a una respuesta ya prevista. La prioridad, en este caso, es formar hábitos automáticos, basándose en una concepción behaviorista del aprendizaje. Después de analizar varias propuestas contenidas en las obras, la autora concluye que, también en obras posteriores a los PCN y producidas por autores integrantes del movimiento de cambio, el trabajo en

la clase de lengua estuvo, en la mayoría de las obras, enfocada en los ejercicios, alejados, muchas veces, de una función social más amplia y de una perspectiva que concibe a los alumnos como sujetos creadores de textos. (Cerqueira, 2010, p. 141)

Para Cerqueira, la enseñanza de la lengua portuguesa necesita ser problematizada, no sólo en el papel que cumple el maestro y el alumno, sino en relación con todos los elementos que constituyen el proceso de enseñanza y aprendizaje. 


\section{Consideraciones finales}

Al abordar los caminos de la enseñanza de la gramática en Brasil, observamos que las concepciones de la gramática se modificaron a lo largo de los años, llegando a diluirse en medio de las actividades del análisis lingüístico. Al mismo tiempo, observamos que parte de los discursos oficiales y académicos proyectan este cuerpo de conocimientos para márgenes de difícil acceso a las producciones del campo didáctico-metodológico, aún inmerso en la polémica con respecto de la pertinencia de si enseñar la gramática, o no. Entre los trabajos analizados, se destaca el esfuerzo para superar el rechazo por la enseñanza tradicional de la gramática. Por lo tanto recurren a los acontecimientos provenientes de diferentes áreas, ya sea de la lingüística, la psicología y/o la investigación educativa.

Por nuestra parte, entendemos que la escuela debe crear condiciones para que los sujetos asuman un control cada vez más amplio de los conocimientos lingüísticos, fundamental para actuar con la lengua en las diversas y complejas situaciones sociales. Sin embargo, los resultados en las evaluaciones a larga escala, las denuncias hechas por los maestros que actúan en los diferentes niveles de enseñanza y los testimonios brindados por los estudiantes se enfocan hacia una misma mirada: las apropiaciones lingüísticas que en la escuela han protagonizado los jóvenes no son suficientes para atender siquiera las demandas propias de la institución escolar tampoco los desafíos de un entorno que les exige estrategias de elaboración, expansión y socialización de los conocimientos.

En este contexto, podemos decir que existe un doble movimiento por ser realizado en relación al estudio de la lengua: uno de ellos va en el sentido de ampliar las situaciones de uso del lenguaje, de manera que el sujeto pueda desarrollar estructuras lingüísticas complexas, utilizadas no solo en la escuela sino también lejos de ella; y el otro movimiento va en el sentido de profundizar y expandir la capacidad fundamentalmente humana de mirar el lenguaje, inquiriéndole, reconociéndole y pensando sobre él, capacidad que el niño usa sólo dentro de la escuela y que es necesario expandir.

Si consideramos que la escuela es una institución al servicio de la humanización del hombre (¡por más redundante que esto pueda parecer!), también es ella uno de los lugares 
apropiados para el ejercicio de la acción intencional sobre los procesos psicológicos, entre los cuales actuar con y sobre la lengua. En qué consiste esto, mediante qué propuestas se realizan y qué efectos produce sobre la formación y la actuación de los sujetos son algunos de los problemas que tienen que ser enfrentados de manera solidaria por las áreas, cuyos conocimientos se ubican en la complejidad que es el aprendizaje siempre inconcluso de la lengua.

\section{Referencias bibliográficas}

Angelo, G.L. de. (2005). Revisitando o ensino tradicional de língua portuguesa. Tese de Doutorado, Universidade Estadual de Campinas, Campinas, SP.

Aparício, A.S. (2010). Modos individuais e coletivos de produzir a inovação no ensino de gramática em sala de aula. Revista Brasileira de Lingüística Aplicada, Belo Horizonte, 10 (4), 883-907.

Bagno, M., \& Rangel, E. de O. (2005). Tarefas da educação lingüística no Brasil. Revista Brasileira de Lingüística Aplicada, 5 (1), 63-81.

Brasil. Secretaria de Educação Fundamental. (1998). Parâmetros curriculares nacionais: Terceiro e quarto ciclos do ensino fundamental: língua portuguesa. Brasília : MEC/SEF. Disponible en: http://portal.mec.gov.br/seb/arquivos/pdf/portugues.pdf, consultada el 24.05.2011.

Brito, C.C. de P. (2011). Discurso(s) sobre o ensino de língua materna em um curso de formação de professores. Revista Brasileira de Lingüística Aplicada, Belo Horizonte, 11 (3), 633-651.

Bronckart, J-P. (1999). Atividades de linguagem, textos e discursos. São Paulo: EDUC.

Bunzen, C. (2011). A fabricação da disciplina escolar Português. Revista Diálogo Educacional, 11 (34), 885-911.

Cerqueira, M.S. de. (2010). Atividade versus exercício: Concepções teóricas e a prática da produção textual no ensino de língua portuguesa. Trabalhos em Linguística Aplicada, Campinas, 49 (1), 129-143.

Culioli, A. (1999). Pour une linguistique de l'énonciation. Formalisation et operations de repérage. Paris: Ophrys. v. 2.

Dolz, J., \& Schneuwly, B. (2003). Gêneros orais e escritos na escola. Campinas, SP: Mercado de Letras.

Fairchild, T.M. (2010). Conhecimento técnico e atitude no ensino de língua portuguesa. Educação e Pesquisa, São Paulo, 35 (3), 495-507.

Faraco, C.A. (1984). As sete pragas do ensino de português. En: Geraldi, J.W. (Org.). (1984). O texto na sala de aula: Leitura e produção. (pp. 17-23), Cascavel: Assoeste.

Geraldi, J.W. (Org.). (1984a). O texto na sala de aula: Leitura e produção. Cascavel: Assoeste. 
Geraldi, J.W. (1984b). Concepções de linguagem e ensino de português. En: Geraldi, J.W. (Org.). (1984). O texto na sala de aula: Leitura e produção. (pp. 41-48), Cascavel: Assoeste.

Geraldi, J.W. (1984c). Unidades básicas do ensino de português. En: Geraldi, J.W. (Org.). (1984). O texto na sala de aula: Leitura e produção. (pp. 49-69), Cascavel: Assoeste.

Geraldi, J.W. (1993). Portos de passagem. São Paulo: Martins Fontes.

Geraldi, J.W. (1996). Linguagem e ensino: Exercícios de militância e divulgação. Campinas, SP: Mercado de Letras.

Gonçalves, A.V., Saito, C.L.N., \& Nascimento, E.L. (2010). A língua em funcionamento nas práticas discursivas. Revista Brasileira de Lingüística Aplicada, Belo Horizonte, 10 (4), 995-1024.

Lobato, M. (1994). Fábulas. São Paulo: Brasiliense.

Marinho, M. (2003). O discurso da ciência e da divulgação em orientações curriculares de Língua Portuguesa. Revista Brasileira de Educação, 24, 126-139.

Perini, M.A. (1985). Para uma nova gramática do português. São Paulo: Ática.

Perini, M.A. (2010). Gramática do português brasileiro. São Paulo: Parábola.

Pietri, E. de. (2010). Sobre a constituição da disciplina curricular de língua portuguesa. Revista Brasileira de Educação, 15 (43): 70-84.

Possenti, S. (1996). Por que (não) ensinar gramática na escola. Campinas, SP: Mercado de Letras: Associação de Leitura do Brasil.

Silva, N.I. da. (2010). Ensino tradicional de gramática ou prática de análise linguística: Uma questão de (con)tradição nas aulas de português. Revista Brasileira de Lingüística Aplicada, Belo Horizonte, 10 (4): 949-973.

Soares, M. (1989). Linguagem e escola: Uma perspectiva social. São Paulo : Ática.

Soares, M. (2004). Português na escola: História de uma disciplina curricular. En: Bagno, M. (Org.) Lingüística da norma. 2.ed. (pp. 155-176), São Paulo: Loyola.

Travaglia, L. C. (1995). Gramática e interação: uma proposta para o ensino de gramática no $1^{\circ}$ e $2^{\circ}$ graus. São Paulo : Cortez.

Unesco. (1990). Declaração mundial sobre educação para todos. Conferência Mundial sobre Educação para Todos: satisfação das necessidades básicas de aprendizagem. Jomtien, Tailândia, 05-09/3/1990. 23 p. Disponible en: http://unesdoc.unesco.org, consultada el 19.05.2006.

\footnotetext{
${ }^{1}$ Los textos citados en este artículo están publicados en portugués. Las traducciones al español son de nuestra responsabilidad.

${ }^{2}$ La educación básica en Brasil se constituye de tres niveles: la educação infantil (de 0 a 5 años), el ensino fundamental (de 6 a 14 años) y el ensino médio. Sólo el ensino fundamental es obligatorio y gratuito.

${ }^{3}$ Con padrón nos referimos a la modalidad culta de la lengua portuguesa, descrita en la gramática normativa.

${ }^{4}$ Un análisis más amplio de las concepciones de los PCN del área de Lengua Portuguesa, se puede ver en Marinho (2003), Bunzen (2011), Aparício (2010), entre otros.

${ }^{5}$ Los PCN de Lengua Portuguesa fueron publicados en dos volúmenes: uno de ellos es dirigido a los Años Iniciales del Ensino Fundamental (EF) y otro a los Años Finales del EF. Los dos textos están fundados en las mismas aproximaciones teórico-metodológicas. Utilizamos para las citas la edición de los PCN de los Años Finales del EF (Brasil, 1998).
} 


\footnotetext{
${ }^{6}$ Geraldi (1993) atribuye los conceptos actividad epilingüística y metalingüística al lingüista francês Antoine Culioli (1999).
}

\section{Referencias de la autora:}

Adriana Dickel es profesora titular de la Universidade de Passo Fundo (Brasil). Es licenciada en Letras por la Universidad de Passo Fundo y realizó sus estudios de Máster y Doctorado en Educación en la Universidad Estadual de Campinas. Forma parte de dos grupos de investigación: GEPALFA - Grupo de Estudos e Pesquisas sobre Alfabetização y GESPE - Grupo de Estudos e Pesquisa em Educação. Desarrolla también tareas en proyectos de formación continua de professores en el contextos socioeconómicos desfavorecidos.

Email: dickel@upf.br

Para citar este artículo:

Dickel, A. (2012). La enseñanza de la gramática en la escuela: lo que piensan, cómo la abordan y qué proponen las investigaciones brasileñas. Bellaterra Journal of Teaching \& Learning Language \& Literature, 5(2), 1-24. 